\title{
Intestinal Malrotation With a Fixed Partial Volvulus in an Adult
}

\author{
Youn Joon Park \\ Department of Pediatric Surgery, Dankook University College of Medicine, Cheonan, Korea
}

\begin{abstract}
A 44-year-old man had been suffering from nausea, vomiting and watery diarrhea for 5 days and was then admitted to Dankook University Hospital. He had suffered from several episodes of mild symptoms, including abdominal distension, loss of appetite, easy satiety, nausea, vomiting, and diarrhea throughout his lifetime, but most episodes had been ignored by him or physicians. An upper gastrointestinal series and a computed tomography scan revealed an intestinal malrotation with a volvulus. In order to untwist the small bowel in a counterclockwise direction to about 180 degrees, we had to perform not only a dissection of Ladd's band, but also a dissection of other adhesions between the mesocolon and the mesenteric vessel trunk. Surgical intervention needs to be performed for an old intestinal malrotation with any symptoms because the structural or morphological change proceeds as time passes, which is caused by fibrosis due to tension being repetitively applied to Ladd's band, leading to its contraction. Furthermore, a severe contraction may even lead to a fixed partial volvulus.
\end{abstract}

Keywords: Intestinal malrotation; Intestinal volvulus

\section{INTRODUCTION}

An intestinal malrotation is a crucial disease because of its fatal complications, such as a midgut volvulus, which needs immediate surgical treatment. Approximately $90 \%$ of the symptoms usually appear within 1 year after birth [1]. In adults, the diagnosis of intestinal malrotation is more difficult than it is in infants because of its rarity and vague symptoms and because knowledge of this disease is lacking. Many published reports have presented its symptoms and have discussed the necessity for surgical correction of an intestinal malrotation in adults $[2,3]$.

The author believes that many case reports and original articles on intestinal malrotations might have missed old duodenal obstructions by Ladd's band, causing contractions that can result in

Received: March 3, 2015 - Accepted: April 14, 2015

Correspondence to: Youn Joon Park, M.D.

Department of Pediatric Surgery, Dankook University College of Medicine,

119 Dandae-ro, Dongnam-gu, Cheonan 330-997, Korea

Tel: +82-41-550-3796, Fax: +82-41-556-3878

E-mail:psurgeon@naver.com

(C) 2015 The Korean Society of Coloproctology

This is an open-access article distributed under the terms of the Creative Commons Attribution NonCommercial License (http://creativecommons.org/licenses/by-nc/3.0) which permits unrestricted noncommercial use, distribution, and reproduction in any medium, provided the original work is properly cited. a chronic fixed volvulus. Therefore, missing points might lead the surgeon to treat a mildly symptomatic intestinal malrotation conservatively and to overlook the necessity for surgical exploration in cases where it is required. We report an experience with an unusual form of a malrotation with a chronic and fixed partial volvulus found in an adult.

\section{CASE REPORT}

A 44-year-old man with a height of $157 \mathrm{~cm}$ and a weight of $44 \mathrm{~g}$, who had been suffering from nausea, vomiting and watery diarrhea for 5 days, was admitted to the hospital. He was runty at first glance. He had suffered from several episodes of mild symptoms, including abdominal distension, loss of appetite, easy satiety, nausea, and diarrhea throughout his life. He occasionally had severe symptoms of vomiting, abdominal cramping pain, and weight loss. He had received several gastroscopic exams for his symptoms, but was told it was only gastritis. After gastric decompression and treatment for moderate dehydration and electro-imbalance, we performed a gastrointestinal (UGI) series and a computed tomography (CT) scan one day after admission. The UGI series revealed a marked dilatation of the whole duodenum and an abrupt obstruction (Fig. 1). The CT scan also showed a huge dilated duodenum and a 'whirling' of the mesenteric vessel trunk 
Volume 31, Number 3, 2015

(Fig. 2). The author attempted laparoscopic surgery, but failed because huge duodenum made identification of the parts of the anatomy difficult. In addition, a thick and closely adhered band between the duodenum and the ascending colon, where inevitable iatrogenic bowel injury had been predicted, interrupted the dissection (Figs. 3, 4). The mesenteric vessel trunk was severely adhered to the mesocolon and caused a narrowing of the mesenteric root. All of the whole mesenteric veins were tortuously en-

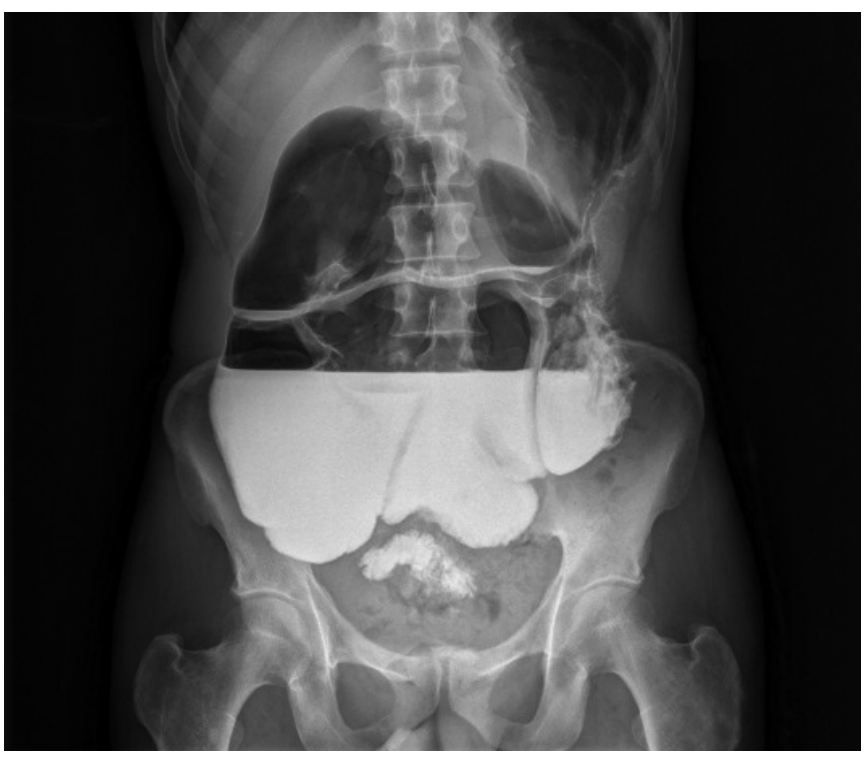

Fig. 1. A upper gastrointestinal series reveals a huge dilated duodenum and minimal passage of gastrografin into the jejunum.

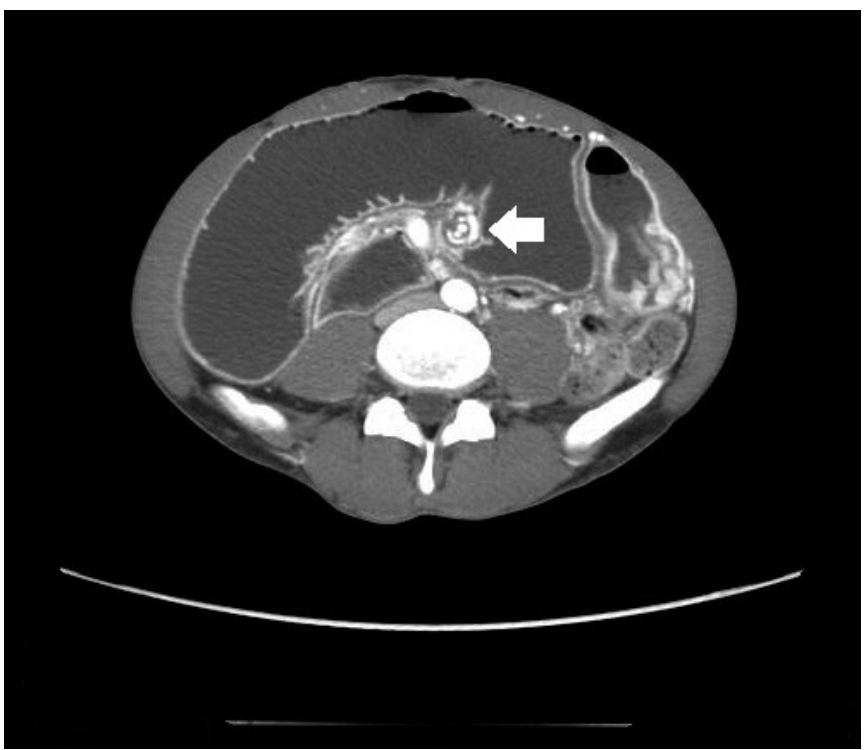

Fig. 2. A computed tomography scan shows not only a huge dilated duodenum but also a 'whirling' of the mesenteric vessel trunk (arrow). gorged by a chronic kink of the proximal veins (Fig. 4). Without relief of the mesocolonic adhesion, a counterclockwise detorsion was not possible. In order to untwist the small bowel by about 180 degrees in a counterclockwise direction, we had to perform not only a simple dissection of Ladd's band but also a dissection of mesocolonic adhesions. At one day after starting oral intake (4 days after surgery), chylous ascites was drained. After conservative treatment, the patient was discharged 19 days after the surgery. At the three month follow-up, he did not feel any relative symptoms.

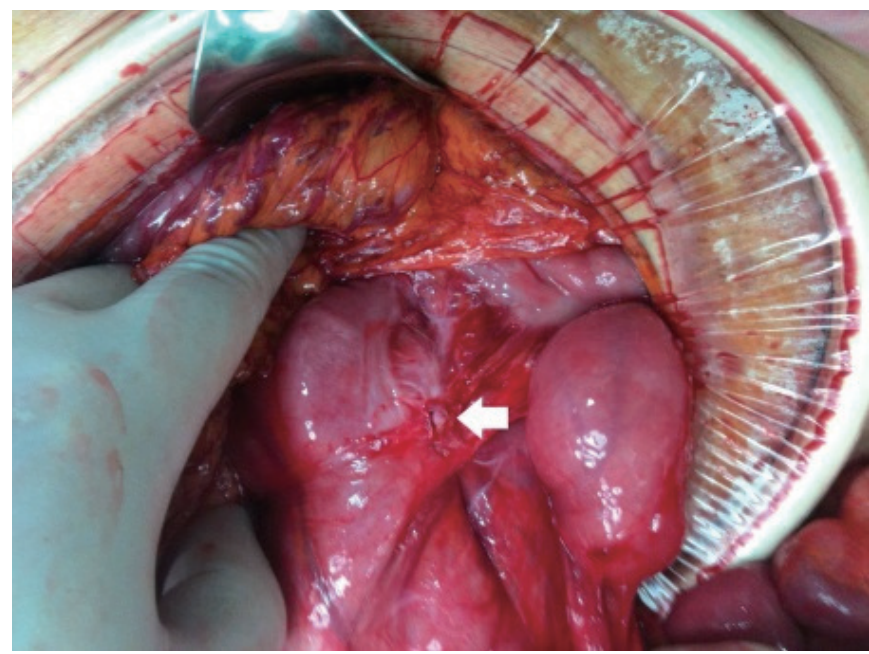

Fig. 3. A dense fibrotic adhesion of the mesocolon and the ascending colon had developed due to recurrent tension (arrow).

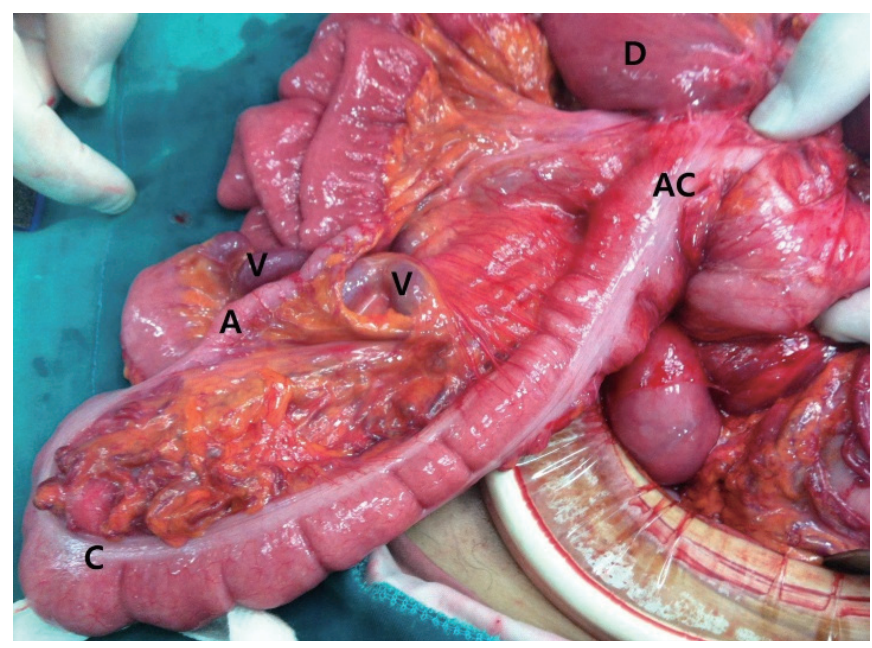

Fig. 4. This photograph shows the duodenum (D), the ascending colon $(\mathrm{AC})$, the cecum $(\mathrm{C})$, and the appendix $(\mathrm{A})$. The relative positions $(\mathrm{V})$ of the duodenum, ascending colon, and engorged mesenteric vessels before the complete dissection between the duodenum (D) and the ascending colon (AC) are shown. 


\section{DISCUSSION}

Intestinal malrotation originates from failure of the normal rotation and fixation of the midgut during the embryonic period. Dott [4] described an intestinal malrotation through embryology, and Ladd [5] reported a corrective procedure for treating an intestinal malrotation. Even after these studies, an intestinal malrotation in an adult was not a disease entity that was familiar to general surgeons because usually surgeons do not fully understand congenital anomalies. Moreover, an intestinal malrotation is not common in adults, and its symptoms are vague and various, ranging from abdominal pain to emesis, nausea, diarrhea, etc. Consequently, some patients undergo an incorrect surgical treatment (gastroileostomy after gastric resection), and some are treated as having a functional or psychiatric disease $[6,7]$. However, in recent days, much knowledge has been accumulated on intestinal malrotations in adults, and the results of many studies have been reported. Especially, the remarkable development of the CT scan allows proper diagnosis and management of this disease.

Nehra and Goldstein [2] described different symptoms of an intestinal malrotation and the incidence of midgut volvulus among three age-related groupings of 170 patients presenting with an intestinal malrotation. According to that study, the most common symptom was abdominal pain in the adult group ( $>18$ years of age), as was emesis in the infant group ( $<1$ year of age). The incidences of midgut volvulus in those two groups were $12 \%$ and $37 \%$, respectively.

Initially, the role of the barium enema was merely to show the cecal position whereas in the 1960s, the UGI series brought a great advancement in diagnosing an intestinal malrotation by directly showing the duodenal-jejunal junction $[8,9]$. Most of all, the development of CT scans make it possible for a quick and precise diagnosis of intestinal malrotations in adults. Through CT scanning, other diseases causing symptoms similar to those of an intestinal malrotation can be eliminated, and an intestinal malrotation in the position relative to the superior mesenteric artery and superior mesenteric vein can be diagnosed [10]. Recently, 3-dimensional CT angiography has allowed the direct diagnosis of a midgut volvulus because of its corkscrew configuration of the mesenteric vessel [11].

Arguments are still ongoing concerning surgical intervention for an asymptomatic intestinal malrotation. Malek and Burd [12] insisted on "watchful waiting" while Durkin and Lund [3] emphasized surgical treatment in all cases of an intestinal malrotation because an unexpected fatal midgut volvulus might occur. Similarly, some agree in surgical treatment because elective surgery has a much lower complication rate than emergency surgery [13]. The author, a pediatric surgeon with much experience in infant intestinal malrotations, tried to approach the treatment of intestinal malrotation by considering the structural or morphologic differences between infants and adults. Many cases of intestinal malrotation in adults are pathophysiologically identical to those of infants, but we experienced a case where the intestinal malrotation had undergone structural and morphological changes through the course of time. In this case, detorsion was not simply achieved by using Ladd's procedure, which is different from infantile cases.

We believe that recurrent events of abdominal symptoms are related to repeated dilation of the duodenum, causing repeated tension to Ladd's band being placed on the duodenum and the ascending colon. The development of dense fibrosis on Ladd's band through tension resulted in traction between the duodenum and the ascending colon. Eventually, a partial volvulus was fixated by the contracted Ladd's band and adhesion of the mesocolon. Consequently, dissection between the duodenum and the ascending colon was difficult due to the adjacent bowels. In such a case, a laparotomy is better than a laparoscopy in the aspects of anatomical confusion caused by the huge dilated duodenum and of unpredictable bowel injury due to the contracted Ladd's band. A chronic fixed volvulus also created tortuous engorgement of the distal mesenteric vein by a mild kink in the proximal vein. Although there was no severe vascular or serosal injury during the surgery, chylous ascites were drained after starting oral feeding. This is also evidence of a chronic fixed volvulus causing lymphatic vessel engorgement. Therefore, even if conservative treatment for an intestinal malrotation may prove to be effective, surgical intervention is still a better method because it eliminates the possibility of continuous morphological change.

Lessin and Luks [14] emphasized the necessity for surgical intervention by placing the word 'asymptomatic' in the debate. The meaning of asymptomatic may be ambiguous both to patients and physicians. Patients may forget they had symptoms as time elapses, patients may not assign much importance to the symptoms, patients may not tell the physician about the symptoms, or physicians simply may not make the connection between the symptoms and the disease. Therefore, we emphasize the need for surgical treatment in all cases of intestinal malrotation in order to reduce any errors caused by misinterpreting 'asymptomatic' and to minimize the chance of morphological change over time after having overlooked mild symptoms. Furthermore, intestinal malrotations presenting with mild symptoms are easily treated with laparoscopic surgery, which is better and safer than a laparotomy.

\section{CONFLICT OF INTEREST}

No potential conflict of interest relevant to this article was reported.

\section{REFERENCES}

1. von Flue M, Herzog U, Ackermann C, Tondelli P, Harder F. Acute and chronic presentation of intestinal nonrotation in adults. Dis Colon Rectum 1994;37:192-8.

2. Nehra D, Goldstein AM. Intestinal malrotation: varied clinical 
presentation from infancy through adulthood. Surgery 2011;149: 386-93.

3. Durkin ET, Lund DP, Shaaban AF, Schurr MJ, Weber SM. Age-related differences in diagnosis and morbidity of intestinal malrotation. J Am Coll Surg 2008;206:658-63.

4. Dott NM. Anomalies of intestinal rotation: their embryology and surgical aspects with report of five cases. Br J Surg 1923;11:251-86.

5. Ladd WE. Surgical disease of the alimentary tract in infants. N Engl J Med 1936;215:705-8

6. Moretz WH. Inadvertent Gastro-ileostomy. Ann Surg 1949;130: 124-36.

7. Gamblin TC, Stephens RE Jr, Johnson RK, Rothwell M. Adult malrotation: a case report and review of the literature. Curr Surg 2003;60:517-20.

8. Houston CS, Wittenborg MH. Roentgen evaluation of anomalies of rotation and fixation of the bowel in children. Radiology 1965; 84:1-18.

9. Berdon WE, Baker DH, Bull S, Santulli TV. Midgut malrotation and volvulus. Which films ar most helpful? Radiology 1970;96: 375-84.

10. Nichols DM, Li DK. Superior mesenteric vein rotation: a CT sign of midgut malrotation. AJR Am J Roentgenol 1983;141:707-8.

11. Bozlar U, Ugurel MS, Ustunsoz B, Coskun U. CT angiographic demonstration of a mesenteric vessel "whirlpool" in intestinal malrotation and midgut volvulus: a case report. Korean J Radiol 2008;9:466-9.

12. Malek MM, Burd RS. The optimal management of malrotation diagnosed after infancy: a decision analysis. Am J Surg 2006;191: 45-51.

13. Moldrem AW, Papaconstantinou H, Broker H, Megison S, Jeyarajah DR. Late presentation of intestinal malrotation: an argument for elective repair. World J Surg 2008;32:1426-31.

14. Lessin MS, Luks FI. Laparoscopic appendectomy and duodenocolonic dissociation (LADD) procedure for malrotation. Pediatr Surg Int 1998;13:184-5. 